\title{
Reconfigurando figuras bidimensionales
}

\section{Reconfiguring two-dimensional figures}

\author{
MARMOLEJO, Gustavo A. ${ }^{1}$ \\ VEGA, Myriam B. ${ }^{2}$ \\ GALEANO, Jorge E. ${ }^{3}$
}

\begin{abstract}
Resumen
Se establece una tipología de comportamientos ante situaciones geométricas que promueven la inclusión de la operación visual de reconfiguración. Su caracterización consideró cinco categorías de análisis: operaciones intra-reconfiguración, cambios de anclaje, dificultades visuales, elementos de control visual y mediación. Los resultados destacan la complejidad en la selección de variables que suscitan la aplicación de la reconfiguración. Como conclusión se establecen pautas para el diseño y aplicación de tareas que susciten reflexiones en torno al estudio de la reconfiguración.
\end{abstract}

Palabras clave: visualización, reconfiguración, elementos de control visual, área

\begin{abstract}
A typology of behaviors is established in geometric situations that promote the inclusion of the visual operation of reconfiguration. His characterization considered five categories of analysis: intrareconfiguration operations, anchor changes, visual difficulties, visual control elements and mediation. The results highlight the complexity in the selection of variables that elicit the application of reconfiguration. In conclusion, guidelines are established for the design and application of tasks that provoke reflections around the study of reconfiguration.

key words: visualization, reconfiguration, control visual.
\end{abstract}

\section{Introducción}

La comprensión de la geometría ocurre mediante la coordinación de actividades de visualización, razonamiento y construcción, cada una con funciones epistemológicas específicas. Si bien el desarrollo ${ }^{4}$ de cada actividad debe transcurrir por separado, la visualización puede privilegiarse como puerta de entrada, soporte e impulso de las restantes (Duval, 1998). La visualización tiene matices y características diferentes según el tipo de representación semiótico considerado (Duval, 2003). El interés de esta investigación recae en la visualización vinculada al registro semiótico de las figuras geométricas bidimensionales.

En este sentido, se asume la visualización como la discriminación de unidades figurales y de las acciones visuales a aplicar sobre ellas, las cuales intervienen, asertivamente o no, al transformar, o intentar transformar, una figura. Para que esta actividad cognitiva sea un recurso que apoye el estudio de la geometría, es indispensable

\footnotetext{
${ }^{1}$ Profesor tiempo completo. Departamento de Matemáticas y Estadística. Universidad de Nariño. E-mai: usalgamav@gmail.com

2 Profesora tiempo completo. Instituto de Educación y Pedagogía. Universidad del Valle. myvega43@gmail.com

3 Profesor tiempo completo. Instituto de Educación y Pedagogía. Universidad del Valle. jorge.enrique.galeano@correounivalle.edu.co

${ }^{4}$ No se entiende en el sentido Piagetiano que alude a cambios estructurales del pensamiento sino en un sentido más amplio de evolución, como la transformación de habilidades cognitivas gracias al ejercicio intelectual propiciado al enfrentar situaciones diseñadas con fines específicos.
} 
reconocerla como una actividad cognitiva subceptible de ser incluida en procesos de enseñanza (Marmolejo \& Vega, 2012). Para hacerlo, han de caracterizarse, entre otros aspectos, los niveles de complejidad cognitiva que suscita la visualización de acuerdo con el tipo de operación visual incluido (Marmolejo, 2014).

La reconfiguración, operación visual de interés en esta investigación, alude a "la división de una figura en subfiguras, en su comparación y en su reagrupamiento eventual en una figura de un contorno global diferente" (Duval, 2017, p. 156). Se considera como la operación de mayor complejidad en el estudio de la geometría (Duval, 2017; Marmolejo \& Vega, 2012; Padilla, 1992; Mesquita, 1989). Esta operación "ha jugado un rol heurístico importante a través de la historia de las matemáticas" (Padilla, 1992, p. 12). Constituye un soporte intuitivo en la demostración de teoremas y en la resolución de problemas (Mesquita, 1989; Padilla, 1992; Sánchez, 2003), entre otros, el Principio de Cavalieri, el cálculo del lado de un cuadrado inscrito en un triángulo rectángulo, la suma de las progresiones indefinidas a) 1/2, 1/4, 1/8,1/16 .... y b) 1, 1/4, 1/16 ..., las relaciones pitágoricas (León, 2005), las fracciones (Freudenthal, 1983), el área (Zacharos, 2006; Kordaki, 2003; Marmolejo \& Vega, 2012; Marmolejo \& González, 2015a).

Como lo indica una exhaustiva revisión de la literatura especializada, son incipientes los trabajos que exploran la pertinencia y necesidad de constituir la reconfiguración como objeto de aprendizaje, en tanto que se trata de una operación cognitiva que, de no orientarse adecuadamente, puede dificultar u obstaculizar el aprendizaje de la geometría. Los pocos estudios, hasta el momento identificados, reportan que para muchos estudiantes su inclusión es compleja e incluso imposible en procesos de comprensión geometríca (Marmolejo \& Vega, 2012), asimismo, que es susceptible de aprendizaje (Mesquita, 1989; Padilla, 1992; Marmolejo y Vega, 2012; Duval, 2017). Pero, no suele ser objeto de reflexión explícita ni en los textos escolares (Marmolejo, Guzmán \& Insuaty, 2015) ni por los educadores de algunos países (Duval, 2017; Inan \& Dogan-Temur, 2010; Marmolejo, Sánchez y Londoño, 2017). Trabajos como los realizados por Mesquita (1989), Padilla (1992), Marmolejo \& Vega (2012), Duval (2017) y Marmolejo \& González (2015b), por su parte, determinan elementos que pueden propiciar o entorpecer la discriminación de una reconfiguración.

Si bien investigaciones como las anteriores aportan elementos de reflexión para comprender el papel de la reconfiguración en el estudio de la geometría, en ningún caso dan respuesta a cuestiones como ¿cuáles son los efectos de incluir en una configuración geométrica, simultáneamente, elementos que pretenden favorecer la discriminación de la operación de reconfiguración? ¿Cuáles tipos o modos de comparaciones entre figuras favorecen u obstaculizan la operación de reconfiguración? ¿Qué puede entorpecer la inclusión de la reconfiguración en una actividad geométrica? ¿Cuáles operaciones intra reconfiguración suscitan mayor complejidad para la discriminación de sus elementos? ¿Cuál es el papel que desempeña la discusión de procedimientos de resolución de tareas en situaciones donde la reconfiguración es la operación pertinente? Cuestiones, unas y otras, determinantes para comprender cómo reaccionan los estudiantes ante la resolución de tareas encaminadas a promover el estudio de la reconfiguración y poder hacer de esta operación visual un apoyo para el estudio de la geometría.

El presente trabajo pretende, pues, aportar elementos que den respuestas tentativas a las cuestiones antes reseñadas. En breve, el propósito de la investigación es establecer una tipología de comportamientos de los estudiantes que aporte elementos de reflexión para el diseño de situaciones de enseñanza donde la reconfiguración sea objeto de atención. La investigación privilegió, de forma implícita, el tópico equivalencia de figuras según sus áreas, frente a otros tópicos presentes en los currículos de matemáticas, pues su estudio exige la transformación de figuras en otras de contorno global diferente, y cumple, como se verá más adelante, con las exigencias para el desarrollo de la aprehensión operatoria: aplicación de modificaciones sobre las figuras, no requiere razonamientos de naturaleza deductiva y no exige construcciones con instrumentos geométricos.

\subsection{Reconfiguración y estructuras de control visual}

Para lograr el propósito propuesto en la investigación se asumió una perspectiva teórica que asume que las figuras geométricas son soportes intuitivos que dotan de sentido y significado el aprendizaje de la geometría. 
Estas coadyuvan en la resolución de un problema o en la búsqueda de una demostración a través de la abducción, consistente en delimitar de entrada la clase de hipótesis o alternativas a considerar. Pero, solo en ocasiones, la interpretación perceptiva cuasi-automática de las figuras coincide con la interpretación matemática pertinente. Hay divergencia en la mayoría de los casos (Duval, 2011; 2017). Por tanto, incluir las figuras como herramientas en la resolución de problemas matemáticos no es obvio ni espontáneo (Duval, 2011, 2017).

La condición previa para la descripción de los procedimientos matemáticos pertinentes en las figuras geométricas es un análisis semiótico relativo a la determinación de las unidades de base constitutivas de este registro, a las posibilidades de su articulación en figuras y a la modificación de las figuras obtenidas; su ejecución, en parte no consciente, permite a las figuras cumplir su función heurística (Duval, 2017). En este sentido, una figura puede dar lugar a aprehensiones de naturaleza diferente (Duval, 1995), las cuales, en algunos casos, se subordinan unas a otras o se relacionan y, en otros, se oponen (Duval, 2003).

La atención del presente estudio recae en la aprehensión operatoria, es decir, en la transformación heurística de las figuras. Son varias las modificaciones posibles que promueven operaciones específicas, que constituyen la productividad heurística de las figuras (Duval, 2017). Las modificaciones mereológicas, en particular, ponen en juego las relaciones existentes entre las partes y el todo. Estas permiten la transformación del contorno global de una figura (Duval, 1995). En estas modificaciones, la reconfiguración constituye la operación de mayor complejidad. Su aporte, para la aprehensión matemática de las figuras es fundamental, pues, suscita el reconocimiento simple de unidades figurales elementales de dimensión 2 en una figura cuando están perceptivamente dominadas por unidades figurales que le son heterogéneas.

El desarrollo de la aprehensión operatoria requiere, en palabras de Duval (2017), la aplicación de tareas cuya resolución exija la introducción de tratamientos figurales así como la solicitud, explícita y sistemática, de aplicación de la operación de reconfiguración. Si bien estas son condiciones necesarias y evidentes, no se consideran en la enseñanza de las matemáticas y, por sí mismas, no son suficientes para asegurar el aprendizaje de la reconfiguración. Es necesario, como mínimo, que las tareas no estén incluidas en actividades de razonamiento que exijan la utilización de axiomas o teoremas y que tampoco requieran cambios dimensionales en la cadena de sub-figuras explicitadas. Además, es indispensable organizar las tareas según el nivel de complejidad visual que subyace a su resolución (de una complejidad menor a una mayor).

En cuanto a la última de las condiciones reseñadas en el párrafo anterior, se ha puesto en evidencia la existencia de estructuras de control a través de las cuales el control de acciones cognitivas se ve influenciado y/o dirigido mediante el aprendizaje y enseñanza de las matemáticas. Estas "permiten expresar los medios necesarios para realizar selecciones, tomar decisiones y promover juicios acerca de si una acción es relevante o no, o si un problema está resuelto" (Balacheff \& Gaudin, 2010, p. 192).

En los estudios sobre la visualización se han identificado estructuras de control visual; aluden a la organización de todo tipo de estrategia o recurso que, explícita o implícitamente, facilita o retarda la consideración y aplicación de acciones visuales (por ejemplo, la reconfiguración) o la comprensión de su papel en la realización, análisis o seguimiento de una tarea matemática (Marmolejo \& González, 2015).

Entre las estrategias y recursos que organizan las estructuras de control visual se encuentran:

- Los factores de visibilidad, es decir, los elementos y estrategias que, incluidos en las figuras, destacan el atributo de forma en una configuración y que suscitan la aplicación de la reconfiguración (Duval, 2017 y Padilla, 1992). Es el caso, de representar la figura de partida fraccionada en las partes a considerar, que haya complementariedad entre las formas de las partes a reorganizar y que una misma sub-figura no deba ser considerada simultáneamente en dos configuraciones que se comparan entre sí. 
- "Los elementos de contraste; líneas resaltadas y punteadas, flechas, introducción de colores, índices, etc." (Marmolejo \& González, 2015b, p. 311).

- "Los enunciados y explicaciones realizadas en lengua natural ...[y]... toda alusión a imágenes que, en uno y otro caso, determinan acciones y/o situaciones que demarcan elementos visuales a privilegiar en el desarrollo o comprensión de la tarea expuesta" (Marmolejo \& González, 2015b, p. 311).

Lo anterior ha permitido identificar cuatro estructuras de control visual, a saber: contenido, procedimiento, visibilidad o iconismo (Marmolejo \& González, 2015b). Las dos primeras consideran, respectivamente, las transformaciones figurales "mostradas" en las definiciones de propiedades y relaciones matemáticas, así como en los despliegues de procedimientos expuestos. La tercera, contempla tanto la inclusión de factores de visibilidad (Padilla, 1992) como de elementos de contraste e índices (Marmolejo \& González, 2015b). El iconismo, por su parte, resalta que la "figura representa o alude a un objeto físico o a una acción física. Las características del objeto o de la acción planteada guían la manera de proceder visualmente" (p. 318). Mientras que las tres primeras estructuras de control visual son pertinentes para el desarrollo de habilidades visuales (por ejemplo, la inclusión de la reconfiguración en el estudio de las matemáticas), el iconismo genera ambivalencias semióticas, cognitivas y contextuales, e introduce dificultades para el desarrollo de la visualización y la construcción de los contenidos matemáticos asociados a ella (Marmolejo, 2014; Galeano, 2015).

Por otra parte, son tres las funciones que desempeña el control visual (función de control visual). Marmolejo (2020) alude a una función simple o disjunta cuando se impone parcial o totalmente, un tipo de visualización específico y pertinente a la resolución de la tarea planteada. Considera la función es por refuerzo cuando son varios los elementos que generan control y que en conjunto imponen una única manera de ver pertinente a la realización o comprensión de la tarea propuesta. Finalmente, asume una función ambigua cuando varios elementos ejercen control sobre el tipo de visualización, pero unos introducen maneras de ver pertinentes para la resolución de la tarea, mientras otros suscitan visualizaciones de naturaleza distinta a las tratadas en el tópico a considerar, las cuales no son pertinentes a la resolución de la tarea propuesta o conllevan a procedimientos engorrosos.

En palabras de Marmolejo (2020), Igual que la mayoría de las estructuras de control, las funciones de control visual son determinantes para inducir el desarrollo de habilidades visuales. Por ejemplo, en primera instancia, un uso mayoritario de tareas en las que la función por refuerzo esté presente, motivaría la reflexión sobre la existencia de operaciones, en consecuencia, permitiría establecer qué procesos visuales son susceptibles de consideración en matemáticas. La realización de tareas que privilegien una función de control simple, pueden considerarse para continuar con la promoción del desarrollo visual [y realizar] su evaluación. Finalmente, es necesario introducir reflexiones que permitan comprender que una misma figura puede desencadenar distintos tipos de visualización, de los cuales, algunos podrían no ser pertinentes o económicos para la resolución de la actividad propuesta, tareas con función ambigua son las que se considerarían para tal fin.

\section{Metodología}

\subsection{Naturaleza de la investigación}

Investigación de carácter cualitativo, interpretativo, descriptivo y básico (Bisquerra, 1989). El instrumento de análisis contempló categorías extraídas del accionar de la población o de investigaciones previas. Los datos se acopiaron a través de las producciones escritas de los estudiantes al resolver las tareas propuestas y de la explicación (oral) de sus formas de proceder. En casos puntuales, para ampliar o precisar los procedimientos descritos, se recurrió a entrevistas semi-estructuradas. La interpretación de los datos consideró el análisis 
funcional propuesto por Duval $(2011 ; 2017)$ para la actividad cognitiva vinculada a los registros semióticos de representación, en particular, lo relacionado con las figuras geométricas y la visualización asociada a ellas.

\subsection{Población, fuentes de datos y trabajo de campo}

La población estuvo constituida por 30 estudiantes con edades entre 10 y 11 años, de tres cursos (10 estudiantes por curso) de grado quinto de primaria (C1, C2 y C3) de igual número de instituciones educativas de la ciudad de Santiago de Cali (Colombia). Los estudiantes fueron seleccionados aleatoriamente y separados del aula (y del resto de estudiantes) donde (y con quienes) habitualmente estudiaban. Ninguno de los estudiantes había participado en propuestas de enseñanza sobre el papel de las figuras en las matemáticas y sus posibilidades operatorias y heurísticas. Todos realizaron las tareas propuestas, por tanto, los datos empíricos recolectados incluyen la totalidad de los procederes de la población seleccionada. En cuanto a las instituciones, se seleccionaron al azar y no existió vínculos entre ellas.

Para explicitar tratamientos básicos de las figuras bidimensionales se propuso un cuestionario con cuatro situaciones. Las características perceptuales de las figuras consideradas en el cuestionario exigen, a quien intenta resolver las tareas propuestas, sobrepasar la aprehensión perceptual (Duval, 2017) o poner en juego una aprehensión operatoria. El objetivo educativo del cuestionario fue suscitar la designación matemática de figuras geométricas (Situación 1) e inducir el estudio del área de figuras planas como un tipo de magnitud: verificación de relaciones de equivalencia y orden (Situaciones 3 y 4) y unión de áreas (Situación 2). No obstante, en las consignas no se hizo alusión explícita a este objeto matemático; se le refirió, implícitamente, a través de la solicitud de transformar figuras o dividir la superficie de una figura en partes no solapadas entre sí.

Así, en la primera situación, se solicitó identificar y designar las sub-figuras que constituyen dos configuraciones geométricas distintas: cuadrados, en una; triángulos, trapecios y paralelogramos, en otra. En la mayoría de los casos, parte o totalidad de la superficie de las sub-figuras están sobrepuestas entre sí. En la segunda situación, la consigna fue fraccionar la superficie de una figura irregular (Figura 1) en cuatro partes: inicialmente, en un triángulo, un rectángulo y dos cuadrados; luego, en un paralelogramo, un trapecio y dos triángulos. En la tercera situación (compuesta por tres tareas, ver cuadro 1), la atención recayó en reconfigurar una figura (Figura 1) en otra mediante la aplicación de una traslación (Figura 2), rotación (Figura 3) o reflexión (Figura 4) sobre una fracción de su superficie, la cual, previamente, debía discriminarse. Finalmente, en la cuarta situación, se debía considerar la composición de acciones visuales previamente reseñadas para resolver un problema de comparación de áreas de superficies planas. La atención de la investigación recae en la tercera de las situaciones descritas.

Para inducir la comparación entre las figuras en cuestión y promover transformaciones, se solicitó explícitamente transformar la figura de partida en la de llegada, cada una representada, independientemente, en una hoja tamaño carta. Las cantidades de área de cada pareja de figuras eran equivalentes. Las superficies estaban representadas sobre un fondo cuadriculado con contornos resaltados (en tono y grosor). Cada pareja de figuras, contaba con partes de los contornos semejantes entre sí (en forma, ubicación y longitud). Los estudiantes tuvieron a disposición, además de las hojas en que las figuras estaban representadas, hojas en blanco donde podían reproducir las figuras y explicitar sus procedimientos, cortar y pegar.

Se solicitó a cada estudiante describir y explicar, paso a paso, de forma escrita los procederes adoptados. Posteriormente, en grupos de tres, y solo para la primera de las tareas, con el fin de precisar la claridad, coherencia y cohesión de las producciones individuales, se leyeron los registros en voz alta, se identificaron salvedades u omisiones que dificultaban la comprensión y seguimiento del proceso referenciado, las cuales se consideraron para mejorar y aclarar la presentación escrita de cada procedimiento. Finalmente, en una puesta 
en común, se explicitaron los procedimientos realizados, se compartieron las descripciones escritas y se identificaron dificultades e inquietudes, tanto particulares como generales.

\section{Cuadro 1}

Situación 3: transformación de una figura en otra de contorno global diferente e igual cantidad de área

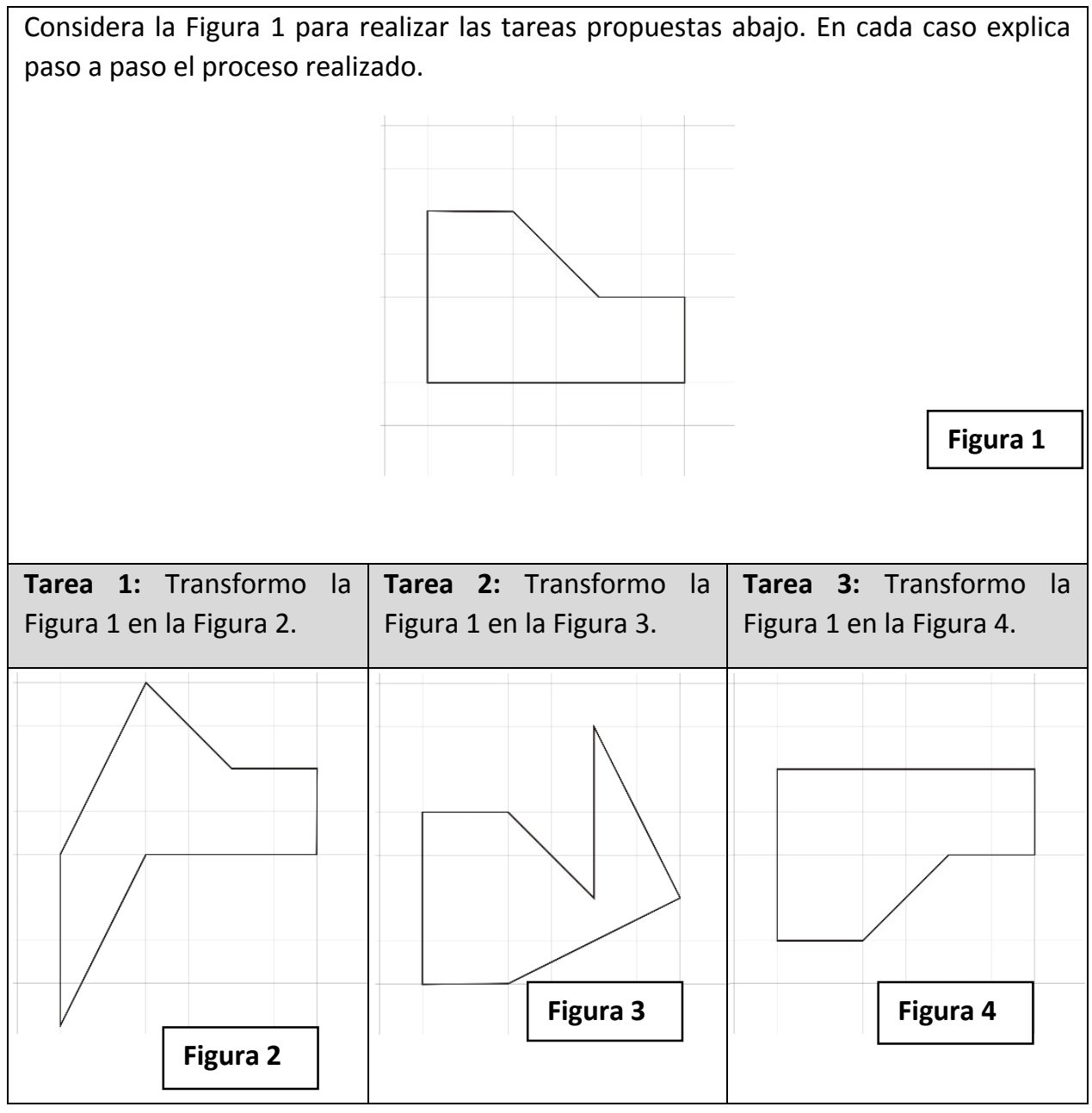

Fuente: Propia

En todos los casos los estudiantes trabajaron con sus profesores habituales, quienes fueron informados en detalle en cuanto a la finalidad de la situación propuesta. La participación del profesor en el trabajo individual, en pequeños grupos y en la puesta en común, se limitó a suscitar la descripción y explicación, en detalle, de las maneras de proceder evidenciadas. Asimismo, en la entrega de tareas, acompañamiento en la lectura de las consignas y recolección de las producciones realizadas. Se emplearon entre 20 y 30 minutos tanto en la resolución y discusión de cada una de las tareas, como en su presentación y re-escritura de los procedimientos presentados.

\subsection{Instrumento de análisis}

La caracterización de los procesos visuales considerados por los estudiantes para reconfigurar las figuras se hizo bajo cinco categorías de análisis: operación intra-reconfiguración, cambio de anclaje, dificultad visual, elemento de control visual y mediación. Las tres primeras aluden a la visualización; las dos últimas consideran, respectivamente, las estrategias incluidas en las tareas que propenden a alentar la consideración de la 
reconfiguración y la manipulación de los soportes en que se representan las figuras en análisis. A continuación, se define cada categoría y se reseñan cómo se describe en la investigación (descriptores).

\subsubsection{Operación intra-reconfiguración}

Acción aplicada a una figura que suscita su reconfiguración en otra de contorno global distinto e igual cantidad de superficie. Fueron seis las operaciones intra-reconfiguración identificados en este estudio, a saber: fraccionamiento, traslación de sub-figuras bidimensionales, rotación de sub-figuras bidimensionales, simetría de sub-figuras bidimensionales, operaciones 1D (composición de acciones sobre partes del contorno de la figura; es el caso de rotación, traslación, fraccionamiento, unión, dilatación, inhibición, etc.) y arrastre (la atención recae sobre los vértices de la figura. Se aplican, sobre ellos, traslaciones y rotaciones. A diferencia de como sucede en los programas de geometría dinámica, la figura de llegada no conserva, en su totalidad, las relaciones geométricas que determinan la figura de partida: el perímetro y la magnitud de los ángulos varían; la forma cambia; solo el área se conserva).

\subsubsection{Cambios de anclaje}

En palabras de Marmolejo \& González (2013) considera los saltos en la focalización de unidades constituyentes de la figura o figuras en análisis, sean de naturaleza bidimensional (sub-figuras o sub-configuraciones), unidimensional (lados) o cero-dimensional (vértices). Los cambios de anclaje explicitado en el estudio fueron: configural ("comparación entre dos o más figuras a partir de sus características globales" (p. 87)), mixto ("exige...considerar simultáneamente las características perceptuales globales e internas de la figura de partida y de llegada" (p. 89)), configural-unidimensional (de considerar las características superficiales globales de las figuras, la atención recae en algunas unidades 1D que constituyen sus contornos. Pone en acto un cambio dimensional operatorio) y Configural-cerodimensional (para centrar la atención en las figuras a comparar se focaliza la atención en sus vértices).

\subsubsection{Dificultad visual}

Acciones visuales o características figurales, que al no ser consideradas o superadas, obstaculizan la realización de la tarea. Se encontró en los procedimientos de los estudiantes tres tipos de dificultades: Ausencia de conservación de la longitud (diferencia entre los perímetros y el número de lados de las figuras de partida y de llegada), Doble composición de cambios de anclaje y fraccionamiento (tras comparar las figuras, se fraccionan sus superficies; luego, la atención recae en una parte de cada una de ellas y se incluye un nuevo fraccionamiento) y Desdoblamiento unidimensional (cuando dos sub-figuras comparten uno de sus lados, una se asume como tal, la otra como una representación unidimensional-abierta, no como una figura bidimensional).

\subsubsection{Elementos de control visual}

"La introducción de ciertos elementos en la figura o la consideración de algunas de sus características, privilegian u obstaculizan unas formas de ver en detrimento de otras" (Marmolejo \& González, 2015b, p. 136). Los elementos de control visual movilizados en el trabajo realizado fueron cuatro, a saber:

- Factores de visibilidad obstaculizantes: dificultan la identificación de la reconfiguración como la operación a considerar. En las tareas se consideran los propuestos por Padilla (1992), es decir, que una misma sub-figura deba ser considerada simultáneamente en dos configuraciones que se comparan entre sí (obstáculo de desdoblamiento) y ausencia de fraccionamiento en partes claves de la superficie de la figura. La investigación, por su parte, aporta evidencias que las similitudes de forma entre dos figuras, al ser una imagen por simetría de la otra, inhibe las posibilidades de considerar la reconfiguración como una operación clave en la resolución de una tarea.

- Factores de visibilidad inductivos: destacan el atributo de forma y suscitan la aplicación de la reconfiguración (Padilla, 1992). En la investigación se incluye que las partes claves a discriminar en la transformación sean 
convexas y que el grado de inclinación permita una fácil identificación de las partes claves. Asimismo, que la forma del contorno de la figura, la presencia de un fondo cuadriculado y un bajo número de operaciones de traslación y rotación a aplicar, promuevan "pistas" para identificar la forma de proceder.

- Elementos de contraste: resaltan atributos de color y textura en la configuración de partida (Marmolejo y Vega, 2012). Promueven la visualización global sobre la local. La primera, acorde al estudio de las matemáticas, la segunda, no. En las tareas se considera la diferencia en tono y grosor entre las unidades 1D que constituyen el contorno de la figura y las que determinan el fondo cuadriculado (Marmolejo \& González, 2015b).

- Procedimiento por referencia: “De manera explícita en la consigna de la tarea, en lengua natural o figuralmente se designan ciertas acciones a aplicar sobre la figura de inicio y/o a los elementos de la figura a considerar, lo cual suscita una manera de ver a privilegiar" (Marmolejo \& González, 2015b, p. 315). Por ejemplo, en lengua natural se alude a la acción de transformar y se representa simultáneamente la forma de la figura de partida y de llegada.

\subsubsection{Mediación}

Acciones aplicadas al papel en que se representan las figuras y suscitan su comparación. Tres elementos describen los tipos de mediación contemplados en la investigación: Superposición (las hojas en que se representan las figuras se ubican una encima de otra, de tal forma que coincidan las partes de sus contornos que son equivalentes, y se comparan entre sí); disyunción (se colocan las hojas en que se representan las figuras, una al lado de la otra; y se procede a compararlas); y recorte (mediante un corte a lo largo de los contornos de la figuras, se extraen sus superficies de la hoja en que se representan; se procede a compararlas).

\section{Resultados}

Las tareas propuestas en la investigación desencadenaron cinco procedimientos visuales de naturaleza diferente: reconfiguración lineal, reconfiguración simple, reconfiguración puntual, reflexión y reconfiguración parcial (simple o lineal). Los primeros permitieron la resolución de la problemática expuesta; no así, en el último de los casos. Estas maneras de proceder destacan la complejidad que subyace a la manipulación de elementos y estrategias que, en los diseños, pretenden promover la inclusión de la reconfiguración. Asimismo, aportan suficientes elementos para reconocer la complejidad de esta operación y establecer pautas para su enseñanza. A continuación, se caracterizan y, en casos puntuales, se reseñan dificultades asociadas a su aplicación; a modo de ejemplo, describimos y representamos fragmentos de las estrategias utilizadas. Iniciamos exponiendo los procederes visuales asociados a la tarea 1 ; luego establecemos comparaciones entre las acciones reseñadas en uno y otro caso; finalmente se expone el proceder de la tarea 3.

\subsection{Procederes visuales asociados a la resolución de la tarea 1}

En la primera tarea, 18 de 30 estudiantes recurrieron a una reconfiguración lineal: estos estudiantes, tras comparar por disyunción las figuras F1 y F2, optaron por un cambio de anclaje configural-unidimensional que suscitó la atención en los lados los contornos de las figuras, sobre ellos se aplicaron operaciones intrareconfiguración 1D. Pero, al ser los perímetros y el número de lados de las figuras distintos, estos estudiantes tomaron los lados como un "material moldeable": aplicaron sobre los lados operaciones que los dilatan o contraen, logrando, así, el objetivo propuesto (Figura 5).

En esta categoría de procedimiento se incluyen los estudiantes que optaron por aplicar: composiciones de rotaciones, dilataciones, fraccionamientos y traslaciones de segmentos (nueve estudiantes); la inhibición e introducción de trazos o sobre partes de ellos (seís estudiantes) y la aplicación conjunta de composiciones de 
rotaciones y traslaciones, fraccionamiento de lados, eliminación de algunos lados y su respectiva unión y reubicación (tres estudiantes).

\section{Figura 5}

Reconfiguración lineal mediante introducción de rotación, fraccionamiento, traslación y dilatación de segmentos

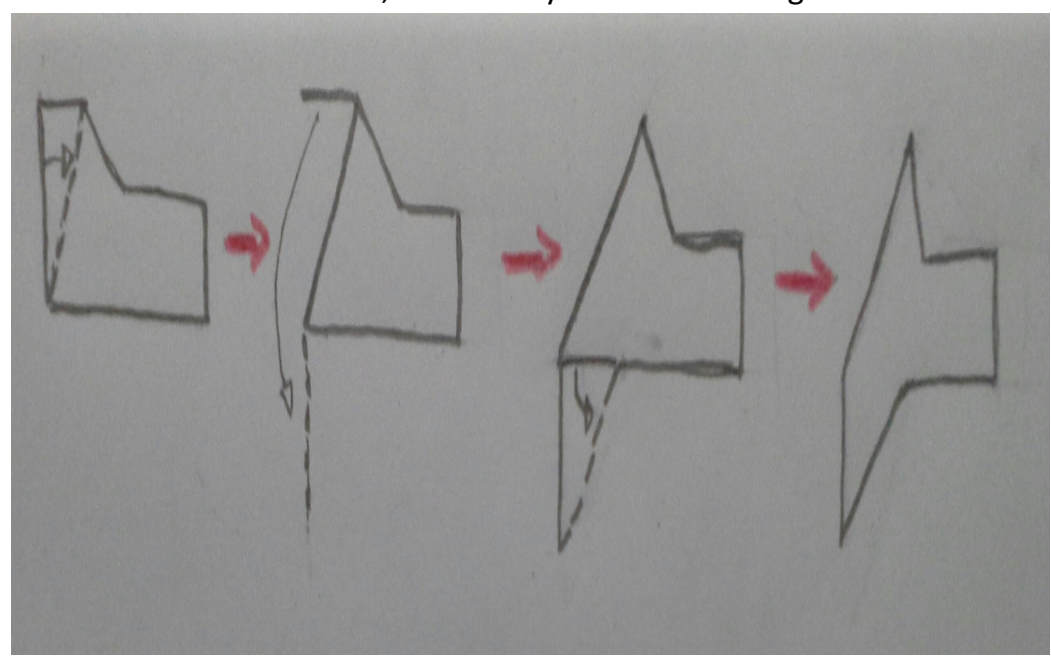

Fuente: Propia

Aplicar la operación de reconfiguración simple fue la opción tomada por cinco estudiantes. Tres compararon las figuras F1 y F2 por superposición, dos por disyunción. En todos los casos, se asumieron dos cambios de anclaje: configural y mixto, uno a continuación de otro. El primero suscitó el fraccionamiento de ambas superficies en dos sub-figuras: triángulo y pentágono; el segundo, permitió la discriminación de la sub-figura a reacomodar (triángulo) y la operación intra reconfiguración (traslación) a considerar (Figura 6).

Figura 6

Reconfiguración simple

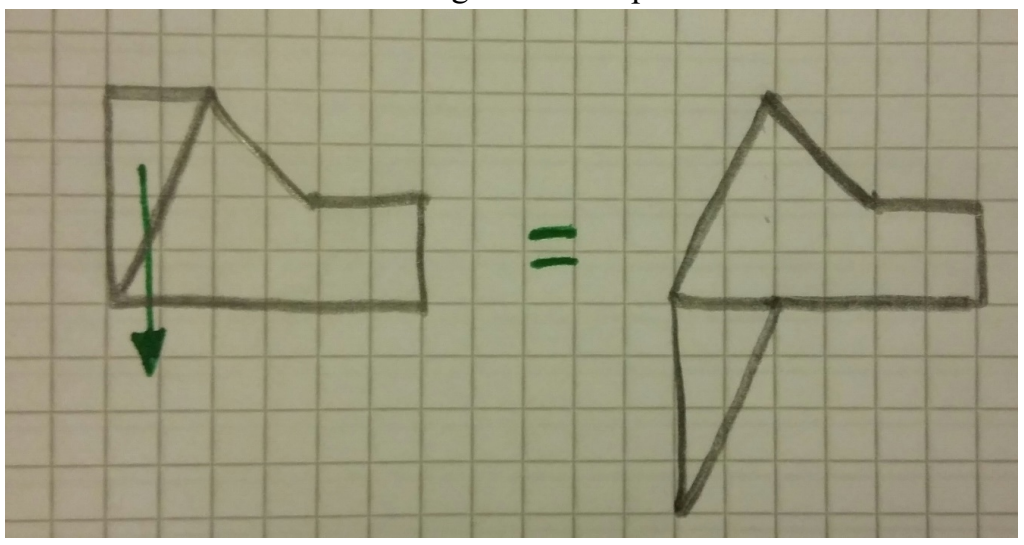

Fuente: Propia

Siete estudiantes optaron por una reconfiguración parcial (lineal o superficial). Seis de ellos, tras comparar por disyunción y considerar un cambio de anclaje configural-unidimensional, reconocieron similitudes y diferencias entre los contornos de las figuras y operaron sobre sus unidades constituyentes. En este caso no se incluyeron operaciones 1D de dilatación o inhibición, solo de traslación y rotación. La tarea no fue resuelta pues "siempre sobraban o faltaban pedazos de lados" (Figura 7).

Figura 7

Reconfiguración parcial lineal 


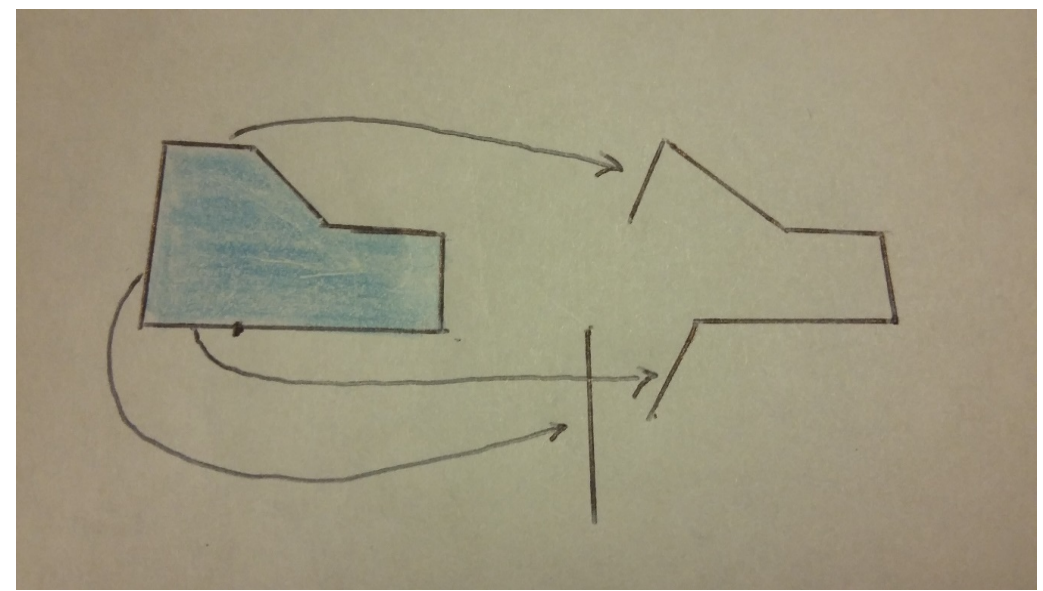

Fuente: Propia

Uno de estos seis estudiantes comparó por superposición. Aplicó un cambio de anclaje configural y fraccionó en dos la superficie de las figuras; en el proceso ninguna representó la sub-figura clave (Figura 8-izquierda). Para identificarla, sería necesario o un nuevo cambio de anclaje (mixto) seguido de un fraccionamiento; o pasar de centrar la atención en las figuras de inicio y llegada a hacerlo en una de las partes en que fueron divididas (S1) e introducir sobre ella un nuevo tipo de fraccionamiento (Figura 8-derecha). Ni una ni otra cuestión fue objeto de consideración.

\section{Figura 8}

Reconfiguración parcial superficial

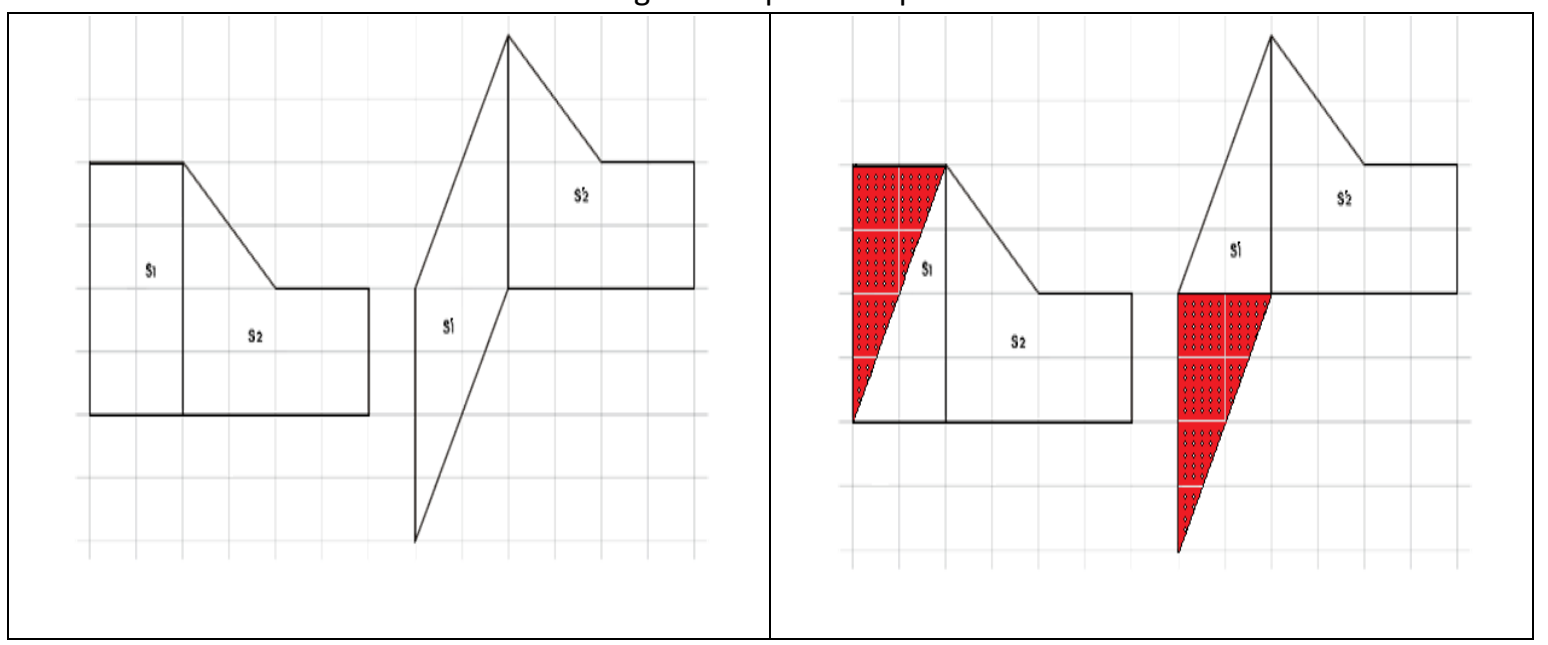

Fuente: Propia

En los dos casos previamente reseñados, tanto la falta de conservación de los perímetros y del número de lados, como la aplicación de una doble composición de cambios de anclaje y fraccionamiento, intervinieron como dificultades que obstaculizaron la realización de la tarea.

Un último estudiante, reconfiguró de forma puntual. Este, tras comparar por disyunción, pasó de un anclaje configural a uno cero-dimensional. En el proceso, discriminó dos tipos de vértices: "comunes" $\mathrm{y}$ "diferentes". Los primeros (BCDEl en la figura F1; y B'C'D'E' $F$ ' en la figura F2); ver Figura 9) determinan las partes de los contornos similares (en cuanto a ubicación en el plano) a las dos figuras; estos se mantienen fijos. Los segundos (A y $\mathrm{F}$ en la figura F1; $\mathrm{H}^{\prime}$ y G'en la figura F2; ver figura 9) han de reacomodarse; para hacerlo aplicó una operación intrareconfiguración de "arrastre". 
Figura 9

Vértices comunes y diferentes considerados en la reconfiguración puntual

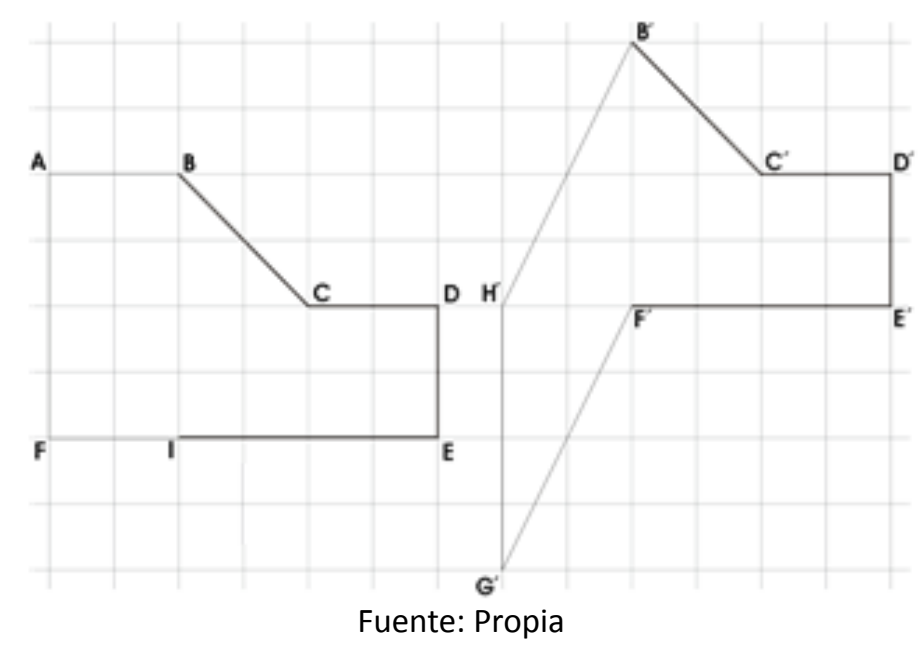

Los resultados anteriores suscitan la siguiente inquietud: ¿Cómo se explica que al intentar transformar una figura en otra, 18 de los 30 estudiantes focalicen espontáneamente su atención en las líneas de los contornos, y apliquen operaciones sobre ellas?

Los intentos de respuesta a tal cuestión han de considerar las características del diseño de la Tarea 1: se introdujeron tres elementos de control, a saber: a) el procedimiento por referencia ("transformo la figura 1 en la figura 2" y representación de la figura de partida y de llegada), b) elementos de contraste (líneas del contorno de las figuras vs las que conforman el fondo cuadriculado) y c) factores de visibilidad inductivos (fraccionamiento, convexidad en la sub-figura clave, similitudes en los contornos de las figuras a comparar).

El propósito al incluir estos elementos de control fue, por un lado, promover la comparación entre la figura de inicio y la de llegada y, por otro lado, favorecer la discriminación de partes comunes y distintas que, al compararse, permitirían detectar tanto la sub-figura a reacomodar como el lugar a ubicarla, en consecuencia,la aplicación de la operación intra-reconfiguración a considerar.

Pero, parece ser que los elementos de contraste suscitaron, de forma inesperada para los diseñadores, un cambio dimensional en la forma de ver que desplazó todo interés operativo en la superficie y lo focalizó, exclusivamente, en sus lados. Lo anterior bien pudo ser enfatizado por la exigencia explícita de transformar una figura en otra; así pues, para estos estudiantes, no quedó más opción que recurrir a acciones unidimensionales para alcanzar o intentar la resolución de la problemática planteada. Esto podría mostrar que el control por refuerzo no siempre favorece las acciones visuales esperadas. Sin embargo, este cambio no debe asociarse a la deconstrucción dimensional (Duval, 2005), la cual se constituye en una forma de ver sobre las figuras que aporta a la comprensión de sus propiedades y a la promoción de una visualización no icónica (Galeano, 2015).

\subsection{Procederes visuales asociados a la resolución de la Tarea 2}

La comparación por superposición y la aplicación de la operación de reconfiguración lineal fueron las formas de proceder más comunes entre los estudiantes al resolver la Tarea 2. La aplicación de la operación de reconfiguración lineal, por su parte, fue la menos considerada. Todos los estudiantes del curso C3 fraccionaron la figura F1 en dos sub-figuras (siendo la sub-figura clave una de ellas). De ellos, ocho centraron la atención en la rotación como la operación intra-reconfiguración a incluir, mientras que dos estudiantes asumieron la composición de operaciones sobre la figura clave. 
Estos resultados contrastan de forma significativa con los descritos en la Tarea 1, donde solo cuatro estudiantes fraccionaron la figura F1 en sub-figuras, pero ninguno resolvió el problema planteado. Los estudiantes que sí lo hicieron, recurrieron a una reconfiguración lineal. La sobreposición física, por su parte, pasó de ser considerada por tres estudiantes en la Tarea 1, a serlo por todos los estudiantes en la Tarea 2.

En cuanto a los estudiantes de los cursos C1 y C2 se observó, también, una disminución en la aplicación de operaciones sobre los lados de la figura F1 y un claro aumento en la aplicación de la operación de reconfiguración simple. En el primero de los casos, de ocho y 10 estudiantes en la Tarea 1 (cursos C1 y C2, respectivamente), se pasó a cinco y seís estudiantes en la Tarea 2 (cursos C1 y C2, respectivamente). En el segundo caso, de dos estudiantes en el curso C1 se llegó a cinco en el curso C1 y a cuatro en el curso C2. Asimismo, sucedió con la aplicación de la operación de superposición en la Tarea 1: solo fue incluida por un estudiante del curso C2. En cuanto a la Tarea 2, la superposición fue contemplada por cuatro estudiantes en cada uno de los cursos.

Es necesario considerar las discusiones tratadas en la puesta en común para comprender por qué el cambió tan abrupto en el proceder de los estudiantes al pasar de la Tarea 1 a la tarea 2. En dicho espacio, los estudiantes expusieron todos los procedimientos de resolución considerados para resolver la Tarea 1.

Los procederes donde se reconfiguró linealmente fueron los primeros en exponerse en el curso C1. En el proceso no se evidenció inconformidad ante las acciones expresadas.

La situación cambió cuando uno de los estudiantes (C1.3) [Para codificar los estudiantes que participaron en la investigación se utilizó una letra y dos dígitos: los dos primeros aluden al curso donde participó el estudiante; el tercero, considera el número que identifica en cada curso al estudiante codificado] comparó por disyunción las figuras en juego e introdujo un trazo que fraccionó la figura F1 en dos sub-figuras (la figura clave y un pentágono irregular), y expresó que "bastaba con dejar caer el triángulo que estaba arriba y colocarlo en la parte de debajo de la figura $1 "$.

Inmediatamente el resto de estudiantes manifestó total rechazo ante tal proceder. Expresaron que "...si el triángulo es todo esto [señalando el contorno del triángulo] y lo bajo... entonces, qué sucede con esta línea de aquí [señalando la hipotenusa del triángulo], la figura de acá [señalando el pentágono] queda abierta". Ante la imposibilidad de encontrar en el grueso de estudiantes argumentos que permitieran justificar y validar este proceder, fue desvirtuada la aplicación de una traslación sobre la figura clave como forma de proceder para la resolución de la tarea. Esto resalta la complejidad que introduce el desdoblamiento al intentar una reconfiguración; asimismo, explica por qué la reconfiguración simple no fue incluida, significativamente, al realizarse la Tarea 1.

Otro estudiante (C1.5) hizo una reconfiguración simple como consecuencia de una comparación por superposición. Sobrepuso las hojas en que se representan las figuras, tal que las partes comunes de sus contornos coincidieran entre sí. Esto llamó poderosamente la atención del resto de estudiantes, pues, la figura F1 "aparecía espontáneamente" fraccionada en un triángulo y un pentágono, por tanto, era posible discriminar, automáticamente, el lugar en que se debía reubicar la primera de las sub-figuras (Figura 10).

Si bien el obstáculo de desdoblamiento también está presente en esta forma de proceder, en este caso no se manifestó inconformidad alguna al respecto. Entonces, ¿discriminar la figura clave de forma "física" y simultánea en dos lugares diferentes es suficiente para asumir que la figura es una representación dinámica, susceptible de reorganización? ¿Esto permitió identificar, de ipso facto, el lugar donde se debe reubicar la subfigura clave y considerar la operación a aplicar? O mejor aún ¿̇la presencia "física" y simultánea de las unidades 1D constituyentes tanto de la sub-figura a trasladar como de su imagen por traslación, constituye, por sí misma, un argumento que permite ignorar o sobrepasar la dificultad que suscita la presencia del obstáculo de desdoblamiento? 
Figura 10

Comparación por superposición y discriminación de la sub-figura clave

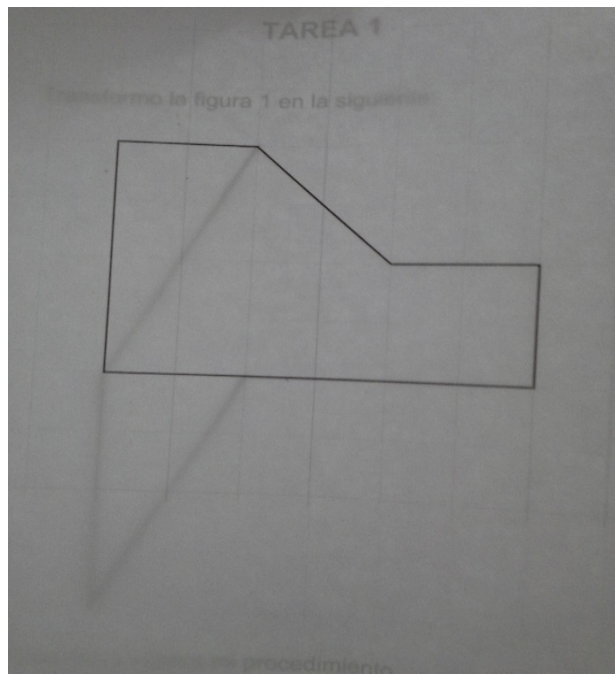

Fuente: Propia

En cuanto a la puesta en común realizada en el curso C2, todos los estudiantes privilegiaron la comparación por disyunción y la reconfiguración lineal. No manifestaron ni dudas ni inquietudes al respecto, tampoco nuevas formas de proceder. Para movilizar discusiones y reflexiones,. En este espacio, uno de los investigadores, presentó los tratamientos explicitados por C1.3 y C1.5, los cuales llamaron poderosamente la atención de los estudiantes por las posibilidades que brindarón tanto para la comparación por superposición como para la reconfiguración simple; ellos. Contrario a cómo sucedió en el curso $\mathrm{C} 1$, los estudiantes no hicieron ningún comentario adicional sobre el proceder de C1.3.

El tratamiento expuestos por C1.3 también fue presentado en la puesta en común realizada en el curso C3. En este caso, igual que sucedió en el curso C1, hubo inquietud y malestar por el proceder de C1.3. Los estudiantes cuestionarón "¿qué pasaba con la parte de la Figura 1 que quedaba sin contorno?”. A continuación, dos estudiantes del curso C3 expusieron la comparación por superposición como forma de proceder. Igual que sucedió en los cursos C1 y C2, causó interés su potencia en la resolución de la tarea, a tal punto que al solicitar la presentación del resto de procederes realizados en el curso, la mayoría (entre ellos siete estudiantes que incluyeron la reconfiguración lineal) manifestó la no necesidad de continuar con las presentaciones, pues en palabras suyas "los métodos presentados eran los mejores para resolver la tarea".

A pesar de la contundente posición de la mayoría de los estudiantes del curso C3, un estudiante (C3.9) insistió en mostrar su procedimiento, él consideró la comparación por superposición pero incorporó una modificación: recortó por el contorno las dos figuras, las comparó por superposición, evidenció diferencias y similitudes entre ellas, y mediante un corte separó la sub-figura clave, luego la trasladó hacia la parte inferior. De esta forma logró la transformación solicitada.

En este caso, en el proceso de recorte, los contornos de las figuras desaparecieron como marcas perceptibles y, por tanto, se inhibió el contraste contorno-superficie. En este sentido, considerar operaciones intrareconfiguración 1D no era factible, pues ya no había lados sobre los cuales operar. No obstante, el corte evidenció dos sub-figuras, las cuales, al separarlas hacen de la traslación, la única operación intra-reconfiguración posible. En este caso, es importante notar que, tras el corte, las superficies en comparación disminuyen, cuestión que en ningún caso fue objeto de discusión. Así pues, en el curso C3 el recorte junto a la comparación por superposición y la reconfiguración simple constituyó la manera de proceder más incluida en la resolución de la tarea 2 . Ningún estudiante de los cursos C1 y C2 optó por este proceder. 


\subsection{Procederes visuales asociados a la resolución de la Tarea 3}

En la Tarea 3 se precisa aplicar sobre la figura F1 una reflexión (sobre la base de F1) o una reconfiguración (Figura 11) para transformarla en la figura F3. Para resolver la tarea propuesta, 23 estudiantes compararon las figuras F1 y F3 por disyunción. De ellos, 19 aplicaron la operación de reflexión y cuatro la operación de reconfiguración lineal. Todos lograron la transformación solicitada. Particularmente, en el primer grupo de estudiantes, se identificó una figura como imagen simétrica de la otra: "las figuras F1 y F3 son la misma figura". Para evidenciar lo anterior, estos estudiantes, primero, aplicaron rotaciones sobre una de las dos figuras (en un sentido y otro, una y otra vez), incluso aplicaron rotaciones, reiterativamente, sobre las dos figuras. En palabras de ellos: esperaban que "la una se convirtiese en la otra". Pero, al percatarse que no lograban el objetivo propuesto, "voltearon" la hoja en que se representa una de las dos figuras, y así lograron resolver la tarea.

Un grupo de siete estudiantes, por otra parte, compararon por superposición las figuas F1 y F3. De ellos, cuatro lograron la transformación solicitada mediante la aplicación de la operación de reconfiguración simple; tres estudiantes identificaron las sub figuras claves pero no lograron aplicar la operación de intra-reconfiguración pertinente (reconfiguración parcial simple).

Figura 11

Reconfiguración por aplicación de

una simetría sobre una sub-figura

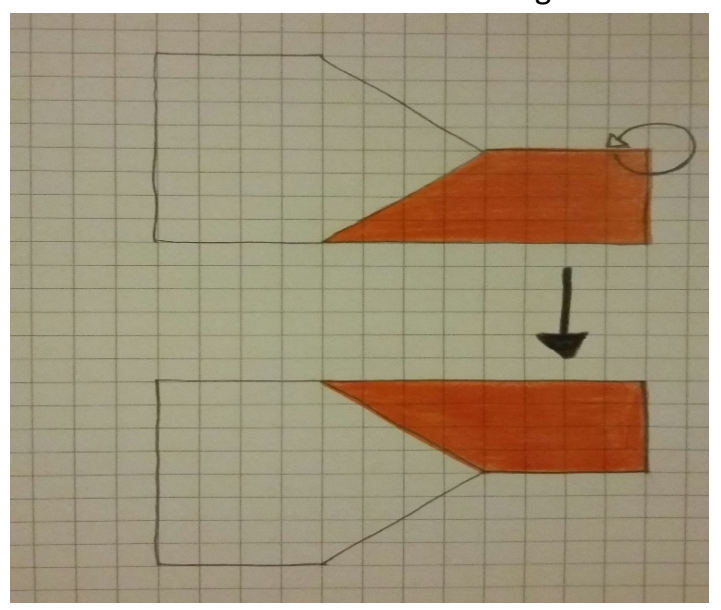

Fuente: Propia

Lo anterior muestra que igual que sucedió en las tareas anteriores, los procedimientos que vinculan la comparación por superposición y la reconfiguración simple fueron los menos considerados. En esta tarea, parece ser que el hecho que una figura sea una imagen por simetría de la otra actuó como elemento de control visual que inhibió la comparación por superposición de las dos figuras, también que la operación de reconfiguración simple fuera objeto de interés. Lo anterior para la mayoría de los estudiantes (19).

Por otro lado, la imposibilidad de realizar la operación de reconfiguración por el grupo de tres estudiantes que sí privilegiaron la comparación por superposición pero que no lograron resolver la tarea, evidencia que la complejidad que subyace para identificar y aplicar una operación de intra-reconfiguración (cuando la sub-figura clave ya ha sido discriminada) depende de la naturaleza de la operación, pues, en las tareas previas, donde la traslación o la rotación eran las operaciones intra-reconfiguración a considerar, todos los estudiantes que discriminaron la sub-figura clave no mostraron inconveniente alguno para alcanzar la reconfiguración en cuestión. Por tanto, en procesos de reconfiguración es más complejo para los estudiantes aplicar operaciones intra-figurales como las simetrías que operaciones visuales como las rotaciones o las traslaciones. 
Para terminar, es necesario señalar que la cuadricula fue un elemento de control que, unido al contraste, hizo que los estudiantes privilegiaran una visualización global sobre una local (Marmolejo \& Vega, 2012). No obstante, en las dos primeras tareas la cuadrícula, en particular, no aportó elementos que apoyaran la reconfiguración como herramienta heurística para la transformación de una figura en otra. Al contrario, su papel pasó a un segundo plano. Bajo la intención, siempre implícita, de controlar que la figura de llegada mantuviese las mismas dimensiones que la figura F1, pasó a ser un instrumento de medida que permitió controlar las longitudes de las líneas operadas, las relaciones existentes entre ellas (en especial la perpendicularidad) y la igualdad entre las cantidades de superficies. En la gran mayoría de los casos, las formas de ver sobre las figuras y las estrategias utilizadas indican que los estudiantes ignoraron su presencia centrando la atención, exclusivamente, en las similitudes y diferencias entre los contornos de las figuras a tratar.

\section{Conclusiones}

Para que los estudiantes ganen en actividades de visualización es importante tener en cuenta que eso no ocurre "de forma inmediata ni simple; más bien es una cuestión de procesamiento de información susceptible de un aprendizaje específico... [que ha de promoverse]...durante los primeros ciclos de la educación básica" (Marmolejo \& Vega, 2012, p. 29). El desarrollo de la visualización exige ganancias en habilidades para la aprehensión operatoria. En este caso, es la operación visual de reconfiguración la que posibilita el desarrollo de tales habilidades (Duval, 2017; Padilla, 1999). Entonces, la reflexión en torno a la operación visual de reconfiguración debe ser propiciada en el aula.

No obstante, en los primeros grados de la educación básica, los currículos escolares dirigidos a la enseñanza de la geometría no promueven su enseñanza (por lo menos de las tres instituciones educativas aquí consideradas). Lo anterior se manifiesta cuando la mayoría de los estudiantes que participaron en la investigación y quienes habían cursado cinco grados de la educación básica, no consideraron la reconfiguración como una operación visual que sucita la resolución de la primera de las tareas propuestas. Para ellos fue objeto de descubrimiento, solo después de ser presentados al grueso de la población los tratamientos figurales de los pocos estudiantes que sí le incluyeron. Lo anterior, unido al hecho de que los estudiantes en una tarea sí y en otra no realizaron actividades de visualización (cuestión reiterativa en la investigación), muestra que la complejidad que subyace a su estudio es alta. Asimismo, que no bastan unas cuantas sesiones de trabajo para el desarrollo de habilidades visuales que aseguren su inclusión.

Los resultados de la investigación aportan elementos puntuales para la cualificación y formación docente, en particular, los relacionados a cómo los educadores pueden promover el desarrollo de la visualización en los primeros grados de la educación básica. Entre variados aspectos, esos resultados permiten comprender el porqué de su complejidad e identifica pautas para el diseño y aplicación de tareas que incluyan actividades de visualización. Entre esas pautas destacamos las siguientes:

- Duval (1998) establece una serie de cuestiones mínimas que habrían de incluirse para alentar el desarrollo de la aprehensión operatoria por parte de los estudiantes. Esta investigación muestra que la variación sistemática de elementos que facilitan o retardan el ver sobre una figura destaca por su complejidad; en particular, resalta la dificultad que representó la predicción de sus efectos.

Lo anterior se ve reflejado en las tareas donde se incluyen elementos de control que por sí mismos, y de forma independiente, favorecen la visibilidad de la reconfiguración, pero que en conjunto tienden a ejercer una función de control ambigua, no de refuerzo. Es el caso de la inclusión de factores de visibilidad inductivos junto a procedimientos de referencia y elementos de contraste; los cuales, en la mayoría de los casos, fueron asociados por los estudiantes a procesos de reconfiguración lineal que obstaculizaron el desarrollo de la aprehensión operatoria (Duval, 1998) y el estudio de magnitudes tipo área y el perímetro, tópicos donde la visualización 
desempeña un papel determinante (Marmolejo, Prada \& Insuasty, 2020; Marmolejo \& González, 2013, 2015a; Kordaki \& Potari, 2002; Outhred \& Michelmore, 1996). Esto, por un lado, suscitó un cambio dimensional en la forma de ver que inhibió la discriminación de sub-figuras y operaciones, y, por otro lado, promovió la concepción según la cual las figuras son las líneas de sus contornos. Este problema está reforzado por prácticas de aula cotidianas, enlas que la designación de las figuras recae en la delineación de sus contornos o la representación de figuras mediante trozos de madera, hilo, palillos, alambres, etc.

- El tipo de comparación entre las figuras de inicio y de llegada, así como la forma en que cada una se representa, se asocian al favorecimiento, obstaculización o inhibición de la función de los elementos de control visual incluidos. La comparación disyunta, por ejemplo, induce la discriminación y operatividad de unidades no pertinentes para la reconfiguración. La comparación por superposición, por su parte, en casos en que las figuras son recortadas y no hay referencia perceptiva a las líneas que conforman sus contornos, produce el efecto contrario; en este caso, el fondo cuadriculado deja de ser un elemento de control.

- La reconfiguración de una figura en otra mediante la aplicación de la operación intra-reconfiguración de reflexión, donde la primera es imagen por simetría (axial) de la segunda, constituyó una tarea de alta complejidad. A primer golpe de vista, la mayoría de estudiantes identificó que las dos figuras eran simétricas entre sí y no vieron la necesidad de reconfigurar. Para ellos, la tarea había sido resuelta mediante esa identificación. No obstante, algunos estudiantes que sí intentaron reconfigurar una figura en la otra, no discriminaron ni la sub-figura ni la operación intra-reconfiguración clave, por tanto, la resolución de la tarea, para ellos, no fue posible. Para los primeros estudiantes, la similitud de formas entre las figuras de inicio y de llegada (como producto de ser una reflexión de la otra), junto a la posibilidad de lograr la transformación pedida bajo la aplicación de dos operaciones de naturaleza distinta (reconfiguración o simetría), constituyó una fuente de obstáculos que impidió discriminar el tipo de reconfiguración a aplicar. Para los segundos estudiantes, asumir la reflexión como operación intra-reconfiguración clave, introdujo una mayor complejidad que la expuesta en tareas donde bastaba con propiciar traslaciones y rotaciones, acciones que en tareas previas fueron objeto de atención.

- La presentación, comparación y discusión de procedimientos de resolución de tareas de visualización, tanto asertivos como no asertivos, fueron una fuente de posibilidades para generar conciencia sobre el papel de la visualización en la geometría, asimismo, para promover procesos de experimentación de procedimientos visuales no considerados por los estudiantes. Fue de esta manera como la mayoría de los estudiantes asumió la reconfiguración simple como proceso posible y pertinente a la resolución de la problemática planteada.

Es clara la importancia de las pautas previas para que los educadores susciten el desarrollo de la visualización en la escuela. Estas consideraciones han de tenerlas en cuenta los educadores sobre todo por la frecuencia con la cual las tareas llevadas al aula no son diseñadas por ellos sino extraídas de pruebas externas y de textos escolares. No sobra insistir en que la visualización va más allá de la reconfiguración (Duval, 1998; AUTOR 1 y XXX, 2013) y que los elementos de control considerados en las tareas propuestas no son los únicos que determinan la visibilidad de todas las operaciones visuales (Padilla, 1998; Marmolejo \& González, 2015b).

En este orden de ideas, para comprender cómo la visualización puede incluirse como objeto de enseñanza, es indispensable asumir nuevas investigaciones que discriminen elementos de control adicionales y sus efectos en la realización de la tarea y, en general, en la aplicación de propuestas de enseñanza (diseñadas por los educadores o propuestas en los libros de textos o mediadas por el uso de TIC), no solo de la operación sobre la cual hemos centrado la atención, sino también para aquellas otras que, por un lado, también promueven la aprehensión operatoria (Duval, 1998; Marmolejo \& González, 2013) y, por otro lado, suscitan su articulación con la aprehensión discursiva o la secuencia de formas (Duval, 1995). 


\section{Referencias bibliográficas}

Balacheff, N. y Gaudin, N. (2010). Modeling Students' Conceptions: The Case of Function. Research in Collegiate Mathematics Education, 16, 183-211.

Bisquerra, R. (1989). Métodos de Investigación Educativa, Barcelona: CEAC. SA.

Duval, R. (2017). Semiosis y pensamiento humano. Registros semióticos y aprendizaje intelectuales (M. Vega Restrepo, Trad.), (2a ed.). Cali, Colombia: Pgrama Editorial Univalle.

Duval, R. (2011). Ver e ensinar a matemática de outra forma. PROEM Editora. Sao Paulo

Duval, R. (2005). Les conditions cognitives de l'apprentissage de la géométrie: développement de la visualisation, différenciation des raisonnements et coordination de leurs fonctionnements. En: Annales De Didactique Et Sciences Cognitives. Volume 10 (pp. 5 - 53).

Duval, R. (2003). Voir en mathématiques. En E. Filloy (Ed.), Matemática educativa. Aspectos dela investigación actual (pp. 41-76). Distrito Federal, México: Centro de Investigación y Estudios Avanzados del IPN.

Duval, R. (1998). Geometry from a cognitive point of view. En C. Mammana y V. Villani (Eds.), Perspectives on the Teaching of Geometry for the 21 $1_{\text {st }}$ Century (pp. 37-51). Dordrecht, Netherlands: Kluwer Academic Publishers.

Duval, R. (1995). Geometrical Pictures: kinds of representation and specific processing. InR. Suttherland y J. Mason (Eds.), Exploiting Mental Imagery with Computers in Mathematics Education (pp. 142-157). Berlin, Germany: Springer.

Freudenthal, H. (1983). Didactical phenomenology of mathematical structures. Dordrecht. Netherlands: Reidel.

Galeano, J. E. (2015). Diseño de situaciones para el trabajo con figuras geométricas basado en las operaciones cognitivas de construcción, visualización y razonamiento. Trabajo de grado Maestría en Educación. Universidad del Valle).

Inan, H.Z. y Dogan-Temur, O. (2010). Understanding kindergarten teacher's perspectives of teaching basic geometric shapes: a phenomenographic research. ZDM. The International Journal on Mathematics Education, 42(5), 457-468.

Kordaki, M. (2003). The effect of tools a computer microworld on student's strategies regarding the concept of conservation of area. Educational Studies in Mathematics, 52(2), 177-209.doi: 10.1023/A:1024065107302.

Kordaki, M. y Potari, D. (2002). The effect of área measurement tools on student strategies: the role of a computer microworld, International Journal of Computers for Mathematical Learning, 7(1), 1-36.

León, O. (2005). Experiencia Figural y Procesos Semánticos para la argumentación en geometría. Disertación doctoral no publicada. Universidad del Valle. Cali, Colombia.

Marmolejo, G-A. (2020). Función de control visual en el tratamiento del área de superficies planas. Un estudio comparativo de libros de texto colombianos y españoles. En Colección Permanente de Publicaciones Docentes de la Facultad de Ciencias Exactas y Naturales de la Universidad de Nariño (1 edición). P. 121. San Juan de Pasto, Gustavo-Adolfo Marmolejo Editor. Editorial universitaria.

Marmolejo G-A. y González, M-T. (2015b). Control visual en la construcción del área de superficies planas en los textos escolares. Una metodología de análisis. Relime, 18(3), 301-328. 
Marmolejo G-A. y González, M-T. (2015a). El área de superficies planas en el campo de la educación matemática. Estado de la cuestión. REIEC, 10(1), 45-57

Marmolejo G-A. y González, M-T. (2013). Visualización en el área de regiones poligonales. Una metodología de análisis de textos escolares. Revista Educación Matemática, 25(3), 61-102.

Marmolejo, G-A., Guzman, L.Y. y Insuasti, A.L. (2016). Introducción a las fracciones en textos escolares de educación básica ¿figuras representaciones estáticas o dinámicas? Revista científica, 23(1), 43-56

Marmolejo, G-A, Prada, R. y Insuasty, E. (2020). La visualización asociada a las figuras geométricas bidimensionales en el estudio de las matemáticas. Una revisión bibliográfica descriptiva entre 1981 y 2016. Espacios, 41(26), 292-307

Marmolejo, G-A., Sánchez, N. y Londoño, S. (2017). Conocimiento visual de los educadores al promover el estudio de la relación perímetro-área. REIEC, 12(2), 18-28.

Marmolejo, G-A. (2014). Desarrollo de la visualización a través del área de superficies planas. Análisis de libros de texto colombianos y españoles (tesis de doctorado). Universidad de Salamanca, Salamanca. España.

Marmolejo, G-A. y Vega, M-B. (2012). La visualización en las figuras geométricas. Importancia y complejidad de su aprendizaje. Educación Matemática, 24(3), 9-34.

Mesquita, A. (1989). L'Influence d'aspects figuratifs dans l'argumentation des élèves en géométrie: éléments pour une typologie. Disertación doctoral no publicada, Université de Strasbourg, Strasbourg. France.

Outhred, L. y Mitchelmore, M. (1996). Children's intuitive understanding of area measurement. En L. Puig y A. Gutierrez (Eds.), Proceedings of the 20 Conference of the International Group for the psychology of Mathematics Education. PME 20, (Vol 4, pp. 91-98). Valencia. España. Universidad de Valencia.

Padilla, V. (1992). L'influence d'une acquisition de traitements purement figuraux pour l'apprentissage des Mathématiques (Thèse de doctorat non publié). Université de Strasbourg, Strasbourg, France.

Sánchez, E. (2003). La demostración en geometría y los procesos de reconfiguración: una experiencia en un ambiente de geometría dinámica. Educación matemática, 15(2), 27-53.

Zacharos, K. (2006). Prevailing educational practices for area measurement and students' failure in measuring areas. Journal of Mathematical Behavior. 25(3), 224-239.

Esta obra está bajo una Licencia Creative Commons

Attribución-NoCommercial 4.0 International

\section{(cc) EY-NC}

